\title{
Anisotropic planar Hall effect in the type-II topological Weyl semimetal WTe 2
}

\author{
Peng Li, ${ }^{1,2,3}$ Chenhui Zhang, ${ }^{1}$ Yan Wen, ${ }^{1}$ Long Cheng, ${ }^{2}$ George Nichols, ${ }^{2}$ \\ David G. Cory, ${ }^{2,4}$ Guo-Xing Miao, ${ }^{2,3, *}$ and Xi-Xiang Zhang ${ }^{1, \dagger}$ \\ ${ }^{1}$ King Abdullah University of Science and Technology (KAUST), Physical Science and Engineering Division (PSE), \\ Thuwal 23955-6900, Saudi Arabia \\ ${ }^{2}$ Institute for Quantum Computing, University of Waterloo, Waterloo N2L 3G1, Canada \\ ${ }^{3}$ Department of Electrical and Computer Engineering, University of Waterloo, Waterloo N2L 3G1, Canada \\ ${ }^{4}$ Department of Chemistry, University of Waterloo, Waterloo N2L 3G1, Canada
}

(Received 31 January 2019; revised manuscript received 22 June 2019; published 20 November 2019)

\begin{abstract}
The giant planar Hall effect arising from a chiral anomaly, and that is related to the Berry curvature, has been predicted but never observed in nonmagnetic type-II Dirac/Weyl semimetals. Here, we report an observation of the anisotropic planar Hall effect in type-II Weyl semimetal $\mathrm{WTe}_{2}$. Interestingly, we observe a chiral-anomaly-induced sinusoidal angular-dependent planar Hall effect when the electric field is parallel to the tilting direction of Weyl cone, i.e., the $b$ axis of $\mathrm{WTe}_{2}$. The planar Hall effect amplitude is linearly dependent on the magnetic fields and decreases gradually as the temperature increases across the topological phase transition temperature. Our observations clearly reveal the footprints on transport from the chiral anomaly feature in type-II Weyl semimetals.
\end{abstract}

DOI: 10.1103/PhysRevB.100.205128

\section{INTRODUCTION}

The Weyl fermions, in condensed matter, refer to the electrons that exist near the linear dispersive band crossing [1]. One of important and unusual phenomenon in Weyl semimetals is the chiral anomaly, which refers to the nonconservation of the number of particles with a given chirality [2]. A negative longitudinal magnetoresistance is usually considered to be a typical feature of Weyl semimetals when the applied magnetic field is parallel to the electric field [3-6]. Compared to the predicted magnetic Weyl semimetal with broken timereversal symmetry, nonmagnetic Weyl semimetals with a broken inversion symmetry, such as TaAs [7], $\mathrm{Na}_{3} \mathrm{Bi}[8,9]$, and $\mathrm{W}(\mathrm{Mo}) \mathrm{Te}_{2}$ [1], have received considerable attention, because the chiral-anomaly-induced negative longitudinal magnetoresistance in the nonmagnetic Weyl semimetals exclude the possibility of spin-dependent phenomena. However, other effects, such as current jetting and the Knudsen effect [10-12], also result in a negative magnetoresistance that prevents the validation of the Weyl nature of materials using transport methods. Nevertheless, the planar Hall effect (PHE) in nonmagnetic Weyl semimetals that we recently proposed can be an indication of the Weyl nature, through transport properties [13-16].

The PHE refers to the Hall resistance when the magnetic field is coplanar with, instead of transverse to the longitudinal and Hall electric fields. In Weyl semimetals, the nontrivial Berry curvature in the momentum space, not the magnetization in magnetic materials, produces the planar Hall resistance $[13,14]$. In type-II Weyl semimetals, the band crossing of electron pockets and hole pockets is tilted. More interestingly, PHE appears to behave quite differently in type-II

\footnotetext{
*guo-xing.miao@uwaterloo.ca

†xixiang.zhang@kaust.edu.sa
}

Weyl semimetals than it does in type-I ones [14]. The planar Hall resistivity in type-I and type-II Weyl semimetals are quadratically and linearly dependent on the magnetic field, respectively [14]. Since the prediction of theoretical work, the giant PHE has been observed in several Dirac/type-I Weyl semimetals, such as $\mathrm{ZrTe}_{5}$, and GdPtBi [17-20]. Although several studies report observing a chiral-anomaly-induced PHE in type-II Dirac/Weyl semimetals, e.g., $\mathrm{VAl}_{3}$ and $\mathrm{WTe}_{2}$ [21,22], the strong orbital magnetoresistance (OMR) in typeII Weyl makes it challenging to know, with certainty, the real physical origin of the observed PHE [23].

In this work, we investigate the anisotropic PHE in type-II Weyl semimetal $\mathrm{WTe}_{2}$, with an electric field along and normal to the tilting direction of the Weyl cone. We find that the planar Hall resistivity, together with the electric field along the tilting direction ( $b$ axis), are linearly dependent on the magnetic field, with an angular dependence of $\sin \varphi$ (where $\varphi$ is the tilt angle between the current and magnetic field), consistently with theoretical predictions. Across the topological phase transition temperature $(\sim 70 \mathrm{~K})$ in $\mathrm{WTe}_{2}$, the amplitude of the chiral-anomaly-induced PHE decrease when increasing the temperature.

\section{RESULTS AND DISCUSSION}

To make the Hall bar devices with a current flow along different directions, we first identify the in-plane crystal orientation of chemical vapor transport grown $\mathrm{WTe}_{2}$ flakes (from HQ graphene, see more details in Supplemental Material Note 1), using polarized Raman spectroscopy. The intensity of the Raman spectra collected with a crystal orientation either parallel or normal to the laser polarization direction are significantly different, especially for the peaks $A_{1}^{8}\left(\sim 130 \mathrm{~cm}^{-1}\right)$ and $A_{1}^{2}\left(\sim 212 \mathrm{~cm}^{-1}\right)$ [24,25], as shown in Fig. 1(a). Through the angular-dependent $A_{1}^{8}$ and $A_{1}^{2}$ peaks, we can identify the 


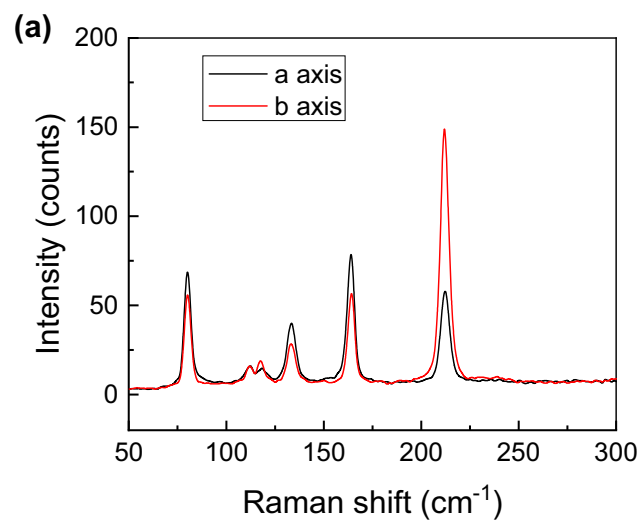

(b)

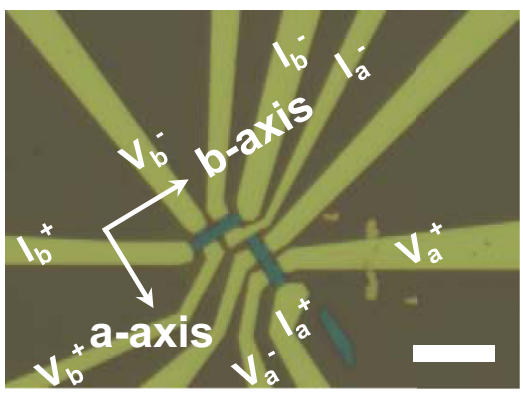

(c)

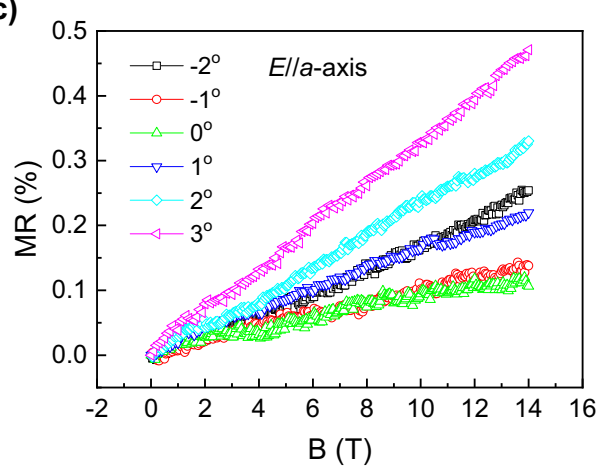

(d)

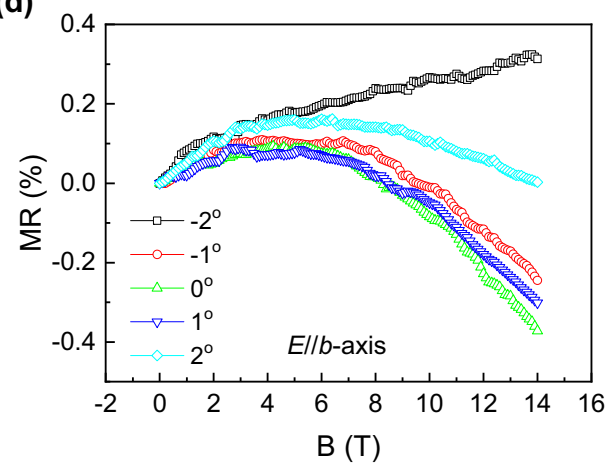

(e)
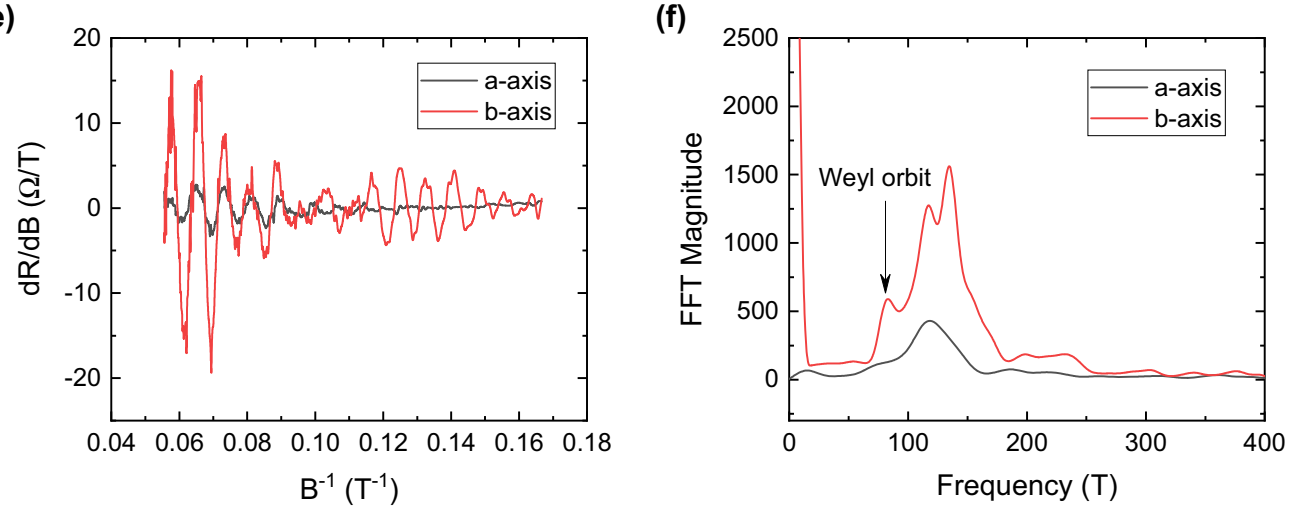

FIG. 1. (a) Typical optical image of $\mathrm{WTe}_{2}$ devices. The scale bar is $10 \mu \mathrm{m}$. The thickness of the device is $18.4 \mathrm{~nm}$. (b) Typical polarized Raman spectra with the laser polarization direction along the $a$ and $b$ axes of $\mathrm{WTe}_{2}$. Magnetic field dependence of longitudinal magnetoresistance at $2 \mathrm{~K}$, with current along the $a$ axis (c) and $b$ axis (d) of $\mathrm{WTe}_{2}$. As the increase of deviation between the current and magnetic field, the negative magnetoresistance in (d) vanishes quickly. (e) Raw SdH oscillations with current along the $a$ and $b$ axes ( $T=30 \mathrm{mK}$ ). (f) FFT spectra of SdH oscillations with current along the $a$ axis (e) and $b$ axis (f) of $\mathrm{WTe}_{2}$. The arrow indicates the Weyl orbit quantum oscillation.

in-plane crystal orientation $[24,25]$ and then fabricate the Hall bar devices (see more details in Supplemental Material Figure S1) [26]. Figure 1(b) shows the optical image of typical WTe 2 Hall bar devices $(18.4 \mathrm{~nm})$, with a current flow along both directions.

According to previous studies [1,5,6,27], the Weyl points in $\mathrm{WTe}_{2}$ sits along the $Y$ axis (b axis). To investigate the chiral anomaly-related phenomena, we must be certain that the Fermi level is close to the Weyl points in our $\mathrm{WTe}_{2}$ devices [6], because the the Weyl points of the bulk $\mathrm{WTe}_{2}$ are $\sim 60 \mathrm{meV}$ away from the Fermi level [1]. We first measure the longitudinal magnetoresistance $(B / / E)$ in both devices. Figures 1(c) and 1(d) show the longitudinal magnetoresistance. Clearly, the magnetoresistance ratio is always positive for the $B / / E / / a$ axis, but negative for the $B / / E / / b$ axis under strong magnetic fields $(B>6 \mathrm{~T})$, and with a quadratic field dependence [28]. As the increase of deviation between the current and magnetic field, the negative magnetoresistance vanishes quickly. Since the chiral Landau levels $(n=0)$ are essential to the negative MR, this observed scenario is consistent with the chiral-anomaly-induced negative magnetoresistance in Weyl semimetals. The chiral Landau levels are missing when $B$ is normal to the Weyl cone tilting direction, but still exist when $B$ is parallel to the Weyl cone tilting direction [29]. According to the calculations on $\mathrm{WTe}_{2}$, the Weyl cone is tilted along the $Y$ direction ( $b$ axis) $[1,27]$. Therefore, a negative longitudinal magnetoresistance is expected [Fig. 1(d)]. The Fermi arc-related quantum oscillations 

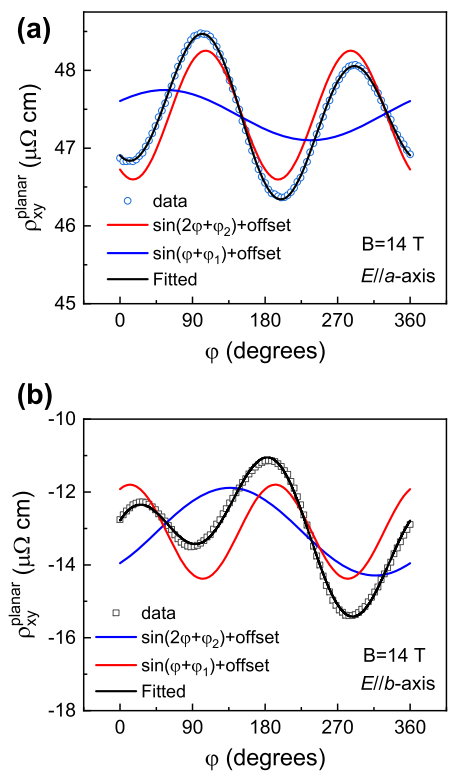
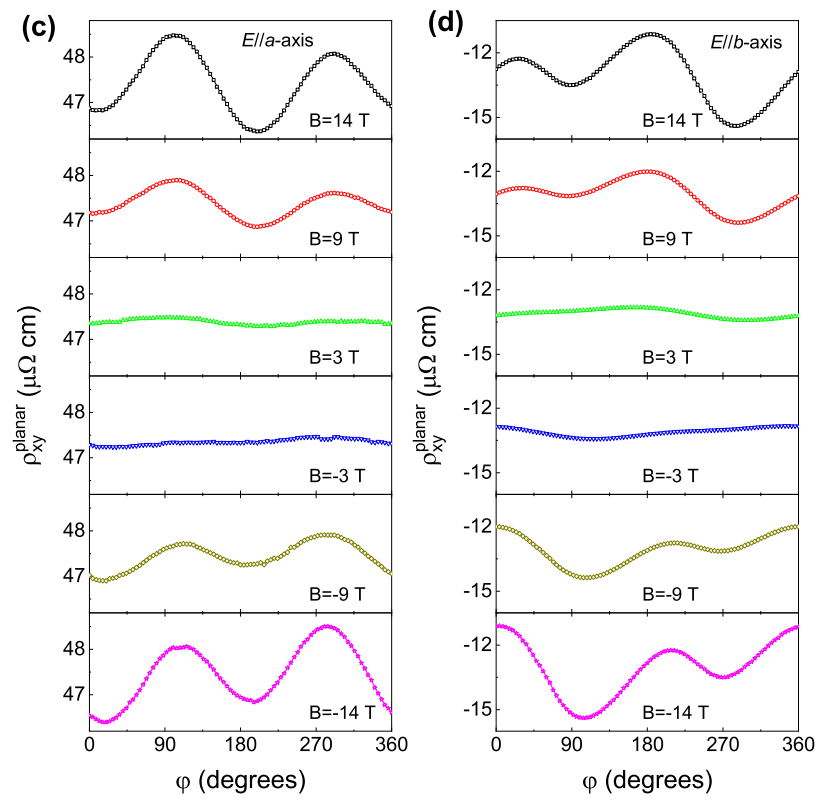

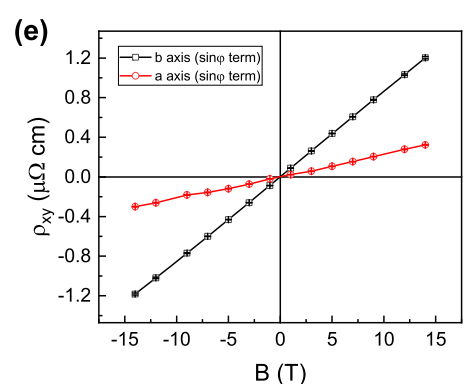

(f)

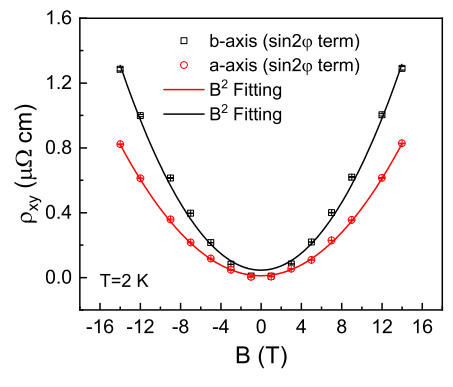

FIG. 2. (a) Angular dependence of planar Hall resistivity of $\mathrm{WTe}_{2}$, with current along the $a$ axis (a) and $b$ axis (b), under a magnetic field of $14 \mathrm{~T}$ and at $2 \mathrm{~K}$. Solid lines give the angular fittings of $\sin \varphi$ and $\sin 2 \varphi$ terms. Raw data of angular dependent planar Hall resistivity at various magnetic fields with current along the $a$ axis (c) and $b$ axis (d). Fitted planar Hall resistivity amplitude of $\sin \varphi$ (e) and $\sin 2 \varphi$ (f) terms as a function of the magnetic field, with current along the $a$ and $b$ axes. Solid lines give the linear and quadratic fitting for $\sin \varphi$ (e) and $\sin 2 \varphi$ (f) terms, respectively.

in flakes with thicknesses no more than the mean free path, the so-called "Weyl orbit," are one more evidence of the location of the Fermi level [6,10,30-33]. According to the Weyl point separation in $\mathrm{WTe}_{2}$, the Weyl orbits should vanish near $\sim 80 \mathrm{~T}$, for a current along the $a$ axis, but remain along the $b$ axis [6]. To uncover the anisotropic Weyl orbits, we measure the quantum oscillations in both devices at $30 \mathrm{mK}$. Figure 1(e) shows the raw data for Shubnikov-de Haas $(\mathrm{SdH})$ oscillations with current along both directions (see more $\mathrm{SdH}$ information in Supplemental Material information Fig. S2). From the fast Fourier transformation (FFT) of the quantum oscillations [Fig. 1(f)], we can readily see the difference between the $a$ and $b$-axis devices. The strong difference in amplitude of $\mathrm{SdH}$ oscillations along both directions comes from the anisotropic mobility [34]. The frequencies near $120 \mathrm{~T}$ are from the bulk electrons' Fermi surfaces of $\mathrm{WTe}_{2}$ [34]. However, we cannot resolve the Weyl orbit frequency $\left(F_{S}=E_{F} k_{0} / e \pi v_{F}\right)$ near $80 \mathrm{~T}$ in the $a$-axis $\mathrm{WTe}_{2}$ device. Nevertheless, the potential Weyl orbit frequency, $\sim 80 \mathrm{~T}$, in the $b$-axis Hall bar, is clearly detected, as indicated by the arrow in Fig. 1(f). These features enable us to further investigate the chiral-anomaly-induced PHE in type-II Weyl semimetals.

Figures 2(a) and 2(b), respectively, give the angular dependent planar Hall resistivity with the current flowing along $a$ and $b$ axes $(B=14 \mathrm{~T})$, where measurements are carried out simultaneously on devices fabricated out of the same flake. Notably, the planar Hall resistivity is composed of both $\sin \varphi$ and $\sin 2 \varphi$ terms. We separate the contributions of $\sin \varphi$ and $\sin 2 \varphi$ terms by fitting the angular dependent planar Hall resistivity, as shown by the solid lines in Figs. 2(a) and 2(b). The fitting equation is

$$
\rho_{x y}^{\text {planar }}=A_{1} \sin \left(\varphi+\varphi_{1}\right)+A_{2} \sin \left(2 \varphi+\varphi_{2}\right)+C,
$$

where $C$ gives the longitudinal resistivity offset due to the Hall bar geometric mismatch. According to previous theoretical studies, the chiral-anomaly-induced PHE for $E$ away from the tilting direction of Weyl cones will give rise to a $\sin 2 \varphi$ angular dependence [14]. At the same time, OMR will generate a $\sin 2 \varphi$ angular dependence in PHE [17,23]. Chiral-anomalyinduced PHE for the case of $E / /$ tilting direction of Weyl cone ( $b$ axis) will also result in a $\sin \varphi$ term [14]. Although the magnetic field is rotating in plane during the measurement of PHE, the slight out-of-plane components of the external magnetic fields will inadvertently introduce Hall signals in the measurements, resulting in a $\sin \varphi$ behavior $[17,19]$. The phase shifts $\varphi_{1}$ and $\varphi_{2}$ in Eq. (1) are necessary to compensate the nonzero initial angle due to the slight misalignment (see more phase angle explanation in Supplemental Material Note 2). According to previous investigations, the carrier density of semimetallic WTe $\mathrm{W}_{2}$ is approximately $10^{19} \mathrm{~cm}^{-3}$ [34], which results in a non-negligible normal Hall effect [26]. Figures 2(a) and 2(b) clearly show that the $\sin \varphi$ term of $E / / b$ axis in the angular dependent planar Hall resistivity is significantly larger than that of the $E / / a$ axis. The simultaneous measurements on devices out of the same flake convince us that the difference of the $\sin \varphi$ term between $E / / a$ axis and $E / / b$ axis configurations should have another physical origin. (See the isotropic normal Hall effect with $E / / a$ axis and $E / / b$ axis in Supplemental Material information Fig. S3).

According to theoretical predictions, in the case of the $E / /$ tilting direction of Weyl cone ( $b$ axis), the planar Hall conductivity $\left[\sigma_{x y}=-\rho_{x y} /\left(\rho_{x x}^{2}+\rho_{x y}^{2}\right)\right]$ will be linearly proportional to the external magnetic field, whereas the planar Hall conductivity will be quadratically dependent on the magnetic field, when $E$ is unparalleled to the tilting direction of the Weyl cone [14]. Figures 2(c) and 2(d) show $\rho_{x y}^{\text {planar }}-\varphi$ curves, 
(a)

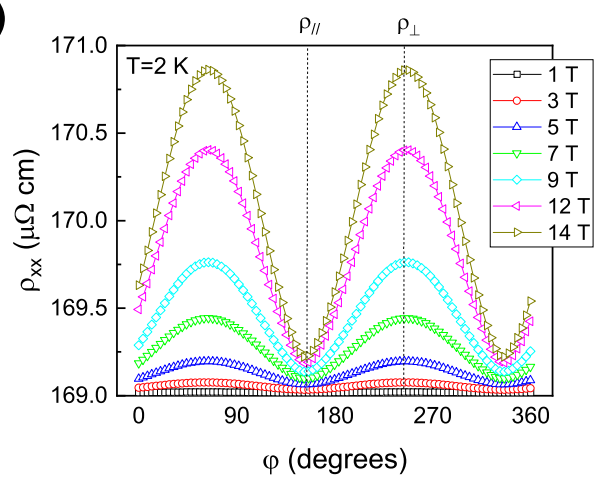

(c)

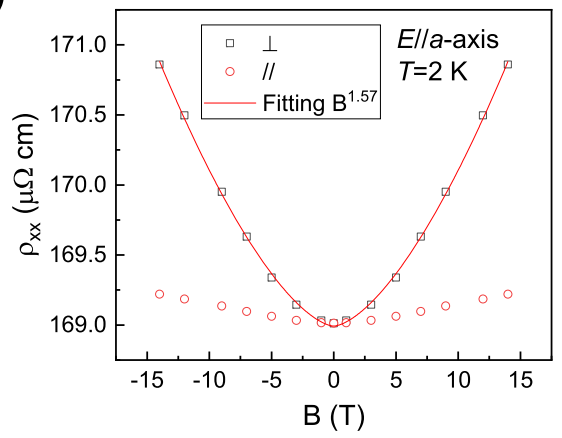

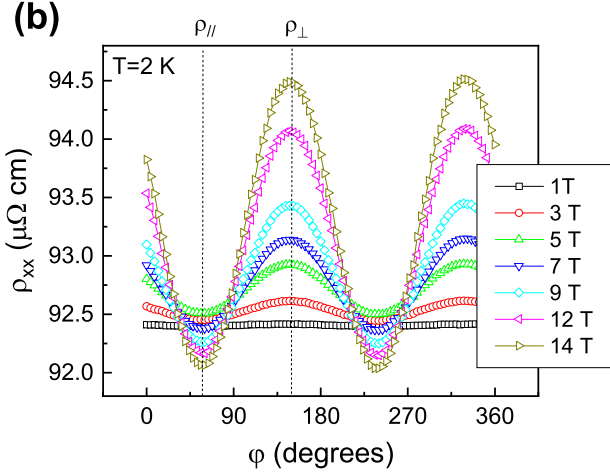

(d)

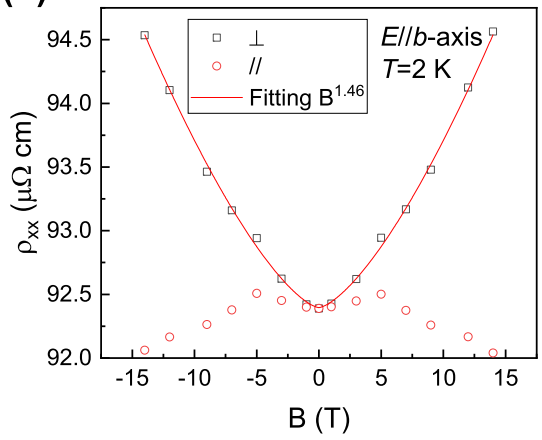

FIG. 3. Measured in-plane anisotropic longitudinal resistivity of $\mathrm{WTe}_{2}(t=18.4 \mathrm{~nm})$, under various magnetic fields, and at $2 \mathrm{~K}$, (a) $E / / a$ axis; (b) $E / / b$ axis. Extracted longitudinal resistivity as a function of the magnetic field, (c) $E / / a$ axis; (d) $E / / b$ axis. The solid lines give the best fittings of $\rho_{\perp}$.

under various magnetic fields $(T=2 \mathrm{~K}$ ), with current along the $a$ and $b$ axes, respectively (see more data in Supplemental Material Fig. S4) [26]. The contributions of $\sin \varphi$ and $\sin 2 \varphi$ terms can be extracted from the raw data using similar sinusoidal fittings. The amplitude of the $\sin \varphi$ term [Fig. 2(e)] linearly depends on the magnetic field for $E$ along both $a$ and $b$ axes. Since the Weyl cone in $\mathrm{WTe}_{2}$ tilts mainly along the $Y$ directions, the $\sin \varphi$ term induced by chiral-anomaly-induced PHE for the $E$ along the $a$ axis is expected to be zero. We infer that the observed $\sin \varphi$ term for $E / / a$ axis in Fig. 2(e) should originate from a normal Hall effect due to out-of-plane magnetic field components. Nevertheless, the $\sin \varphi$ term for $E / / b$ axis is almost four times greater than that for $E / / a$ axis, strongly indicating the existence of a chiral-anomalyinduced PHE in type-II Weyl semimetal $\mathrm{WTe}_{2}$ when $E / /$ tilting direction of Weyl cones ( $b$ axis).

Both OMR and chiral-anomaly-induced PHE for $E$ away from the tilting direction of Weyl cones will follow the $\sin 2 \varphi$ angular dependence on the planar Hall resistivity. Meanwhile, the magnetic field dependence of the $\sin 2 \varphi$ term is approximately quadratic $[14,23]$. Figure $2(f)$ clearly shows that the amplitude of the $\sin 2 \varphi$ term, with current along the $a$ axis, is only smaller by one third than that along the $b$ axis. Ideally, the $\sin 2 \varphi$ term, with current along the $b$ axis, should be zero, if only the chiral-anomaly-induced PHE contributes to the measured signals. This indicates that the OMR presumably dominates, in this highly anisotropic $\mathrm{WTe}_{2}$ [23]. At the exception of the highly anisotropic carrier mobility [34], the nonlinear Hall effect in this highly anisotropic crystals were also demonstrated by second harmonic generation, which is driven by the broken inversion symmetry and the Berry curvature dipole [35-37].

To further demonstrate the existence of a significantly strong OMR in $\mathrm{WTe}_{2}$, we directly measure the angular dependence of longitudinal resistivity, with current along the $a$ and $b$ axes, as shown in Figs. 3(a) and 3(b). Notably, all the $\rho_{x x}-\varphi$ curves $(T=2 \mathrm{~K})$ follow the $\sin 2 \varphi$ relation, no matter in which direction the current flows. The unexpected feature is the $\sin 2 \varphi$ dependence, with current along the $b$ axis. According to the chiral-anomaly-induced PHE theory in type-II Weyl semimetals, the $\rho_{x x}-\varphi$ curve will have a $\sin \varphi$ dependence for $E / /$ tilting direction of Weyl cone [14]. However, we can hardly see the $\sin \varphi$ feature in the curves with the $E / / b$ axis [Fig. 3(b)]. We speculate that the $\sin 2 \varphi$-dependent OMR in $\mathrm{WTe}_{2}$ probably is dominant in the longitudinal resistivity. To uncover more details, we plot the longitudinal resistivities $\rho_{\|}$and $\rho_{\perp}$ as a function of the magnetic field $(T=2 \mathrm{~K})$, as shown in Figs. 3(c) and 3(d), respectively. Clearly, $\Delta \rho=$ $\rho_{\perp}-\rho_{\|}$mainly originates from the enhancement of $\rho_{\perp}$, as the magnetic field increases. In both cases, the field dependence of $\rho_{\perp}$ can be fitted to a power law function, i.e., $\rho_{\perp} \propto B^{1.57}$ and $\rho_{\perp} \propto B^{1.46}$ for the $E / / a$ and $E / / b$ axes, respectively. Both MR follow subquadratic field dependence [38], and the power exponents are quite close to those observed in other systems $[20,21,23]$. Previous work showed that the mobility in $\mathrm{WTe}_{2}$ was highly anisotropic [34], resulting in a highly anisotropic conductivity $(\sigma=n e \mu)$ and a strong anisotropic magnetoresistance in $\mathrm{WTe}_{2}$. This dominant OMR $(\sin 2 \varphi)$ could account for the absence of chiral-anomaly-induced anisotropic MR $(\sin \varphi)$ in the longitudinal resistivity of $\mathrm{WTe}_{2}$. 
(a)

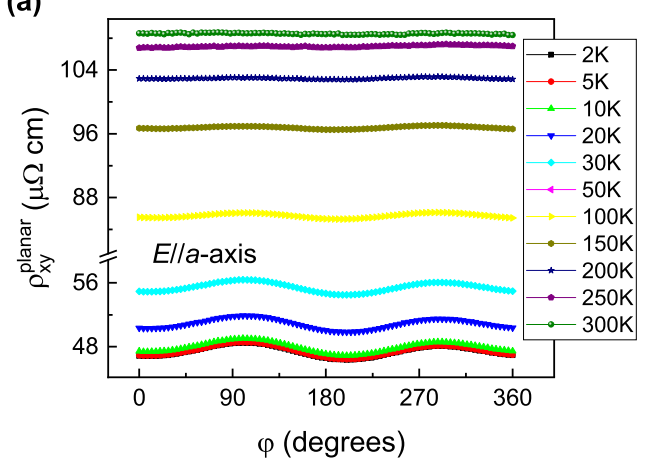

(c)

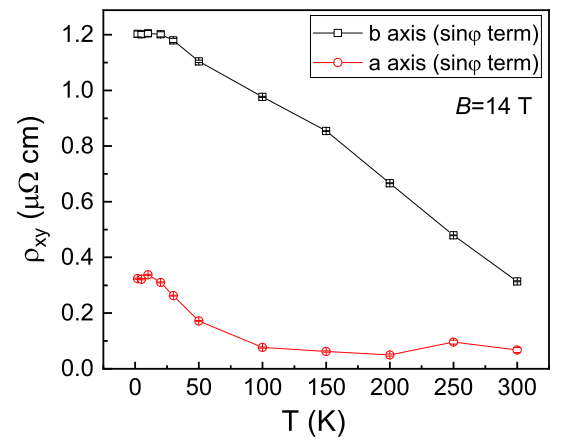

(b)

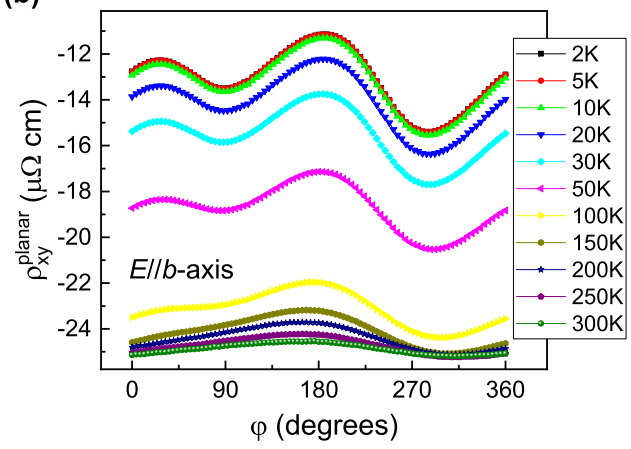

(d)

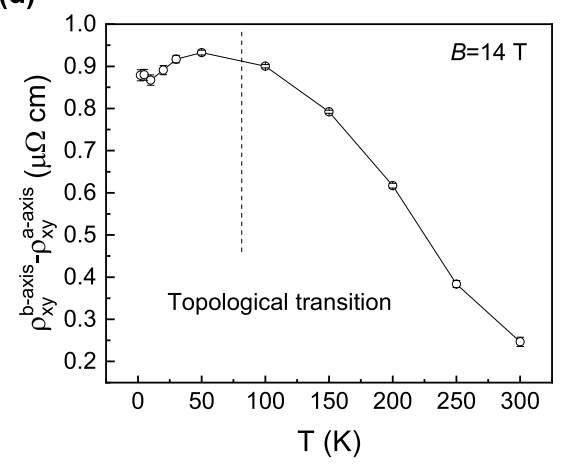

FIG. 4. Measured angular dependence of planar Hall resistivity of $\mathrm{WTe}_{2}$ at different temperatures ( $\left.B=14 \mathrm{~T}\right)$, (a) $E / / a$ axis; (b) $E / / b$ axis(c) Temperature dependence of planar Hall resistivity amplitude $\left(\sin \varphi\right.$ term $\left.\rho_{x y}^{\text {chiral }}\right)$ in $\mathrm{WTe}_{2}$ with current along different directions. (d) Temperature dependence of planar Hall resistivity amplitude difference between the $b$ axis and $a$ axis. It decreases for temperature higher than $100 \mathrm{~K}$.

After observing a linear field dependence of the planar Hall resistivity, we felt motivated to investigate PHE in $\mathrm{WTe}_{2}$ at different temperatures, to further elucidate the physical origin of the $\sin \varphi$ term in the $b$-axis device [Fig. 2(e)]. Figures 4(a) and 4(b) show the angular- dependent planar Hall resistivity, with current along both directions, at different temperatures $(B=14 \mathrm{~T})$. Using the sinusoidal fitting shown in Figs. 2(a) and 2(b), we extract and subsequently plot the temperature dependence of the $\sin \varphi$ - term amplitude in the planar Hall resistivity, along both directions [Fig. 4(c)]. The $E / / a$ axis component almost stays unchanged for $T>100 \mathrm{~K}$, whereas the $E / / b$ axis component decreases continuously. However, the $\sin \varphi$-term component of the $E / / b$ axis is always greater than that of the $E / / a$ axis. We subtract the $E / / a$ axis data from $E / / b$-axis data to eliminate the influence of the outof-plane Hall effect, as shown in Fig. 4(d). The $\rho_{x y}^{b}-\rho_{x y}^{a}$ should be directly related to the highly anisotropic chiral anomaly in type-II Weyl semimetals because of the Fermi arc in $\mathrm{WTe}_{2}$ being mainly along the $Y$ axis. Most strikingly, we find that $\rho_{x y}^{b}-\rho_{x y}^{a}$ remains largely unchanged for $T<100 \mathrm{~K}$, but decreases continuously as the temperature increases up to $100 \mathrm{~K}$. The critical temperature $(50-100 \mathrm{~K})$ in Fig. 4(d) is consistent with the topological phase transition temperature in $\mathrm{WTe}_{2}$ (chemical vapor transport $\mathrm{WTe}_{2}: \sim 70 \mathrm{~K}$ ) previously reported, due to the slight expansion of the lattice [39]. For temperatures above $70 \mathrm{~K}$, the Weyl cone is gapped, and the Fermi arc and chiral anomaly in $\mathrm{WTe}_{2}$ disappear gradually, resulting in a reduction of the chiral-anomaly- induced PHE in $\mathrm{WTe}_{2}$. To further validate our observations and analysis (Fig. 4), we measure more devices of different thicknesses $(t=14.4,27.0 \mathrm{~nm})$ [26]. Both samples show the same features as those reported in Figs. 2 and 4, i.e., a planar Hall resistivity of the $E / / b$ axis higher than that of $E / / a$ axis, and a critical transition temperature within the range of 50-100 K [39]. Our observations demonstrate the presence of a chiral-anomaly-induced $\sin \varphi$ angular dependent PHE in type-II Weyl semimetals, for the E//tilting direction of Weyl cones. On the contrary, one may notice that it is very difficult to extract the $\sin \varphi$ term MR with the $E / / b$ axis, which could be ascribed to the fact that the longitudinal anisotropic magnetoresistance (AMR) ratio is highly anisotropic for current along $a$ and $b$ axes. When the magnetic field of $14 \mathrm{~T}$ is rotating in the $a b$ plane (Fig. 3), the AMR ratios $\left[\left(\rho_{\max }-\rho_{\min }\right) / \rho_{\min }\right]$ with current along the $a$ and $b$ axes are $1.1 \%$ and $2.6 \%$, respectively, indicating clearly the anisotropy. Thus, we cannot use the same subtraction method to extract the $\sin \varphi$ angular dependent MR, induced by the chiral anomaly, from the measured planar Hall effect. In a theoretical work by Nandy et al. [14], only the anisotropic longitudinal MR induced by chiral anomaly was estimated by ignoring the strong orbital magnetoresistance. Actually, this strong orbital magnetoresistance and anisotropic AMR ratio hinders us detecting and extracting the $\sin \varphi$ term MR with $E / / b$ axis in longitudinal configuration $\left(\rho_{x x}\right)$.

Compared to the previous PHE work in other type-II Weyl semimetal systems, i.e., $\mathrm{VAl}_{3}$ and $\mathrm{W}(\mathrm{Mo}) \mathrm{Te}_{2}$ [21-23], we 
analyzed PHE in more detail with the electric field along and normal to the tilting direction of Weyl cone. Their works did not give the clear direction of current in samples. Normally, bulk $\mathrm{W}(\mathrm{Mo}) \mathrm{Te}_{2}$ has a needle shape along the $a$ axis [34]; therefore, we can speculate that, in bulk $\mathrm{W}(\mathrm{Mo}) \mathrm{Te}_{2}$, the current is applied along the $a$ axis, resulting in a $\sin 2 \varphi$-angulardependent PHE without any type-II Weyl feature [14]. On the other hand, the strong OMR also prevents the observation of $a \sin \varphi$-angular-dependent PHE [23]. In our experiments, we carefully define the direction of the electric field, and find our observations in $\mathrm{WTe}_{2}$ to agree with predictions for type-II Weyl semimetals.

\section{CONCLUSIONS}

To conclude, we observed a highly anisotropic PHE in type-II Weyl semimetal $\mathrm{WTe}_{2}$. When $E$ is parallel to the tilting direction of the Weyl cone of type-II Weyl semimetals, the planar Hall resistivity is linearly dependent on the magnetic field, and the PHE decreases fast, for temperatures over the topological phase transition. Taken together with our observations of a chiral-anomaly-induced longitudinal negative magnetoresistance and Weyl orbit quantum oscillations, our results shed light on the chiral anomaly of transport in type-II Weyl semimetals, and its underlying mechanisms.

\section{ACKNOWLEDGMENTS}

The research reported in this publication was supported by King Abdullah University of Science \& Technology (KAUST) with Grants No. CRF-2015-SENSORS-2708 and No. URF-2015-2549 and by the Natural Sciences and Engineering Research Council of Canada (NSERC) Discovery Grant No. RGPIN-04178, the Ontario Early Researcher Award, and the Canada First Research Excellence Fund.
[1] A. A. Soluyanov, D. Gresch, Z. J. Wang, Q. S. Wu, M. Troyer, X. Dai, and B. A. Bernevig, Nature (London) 527, 495 (2015).

[2] H. B. Nielsen and M. Ninomiya, Phys. Lett. B 130, 389 (1983).

[3] X. Huang, L. Zhao, Y. Long, P. Wang, D. Chen, Z. Yang, H. Liang, M. Xue, H. Weng, Z. Fang, X. Dai, and G. Chen, Phys. Rev. X 5, 031023 (2015).

[4] Q. Li, D. E. Kharzeev, C. Zhang, Y. Huang, I. Pletikosic, A. V. Fedorov, R. D. Zhong, J. A. Schneeloch, G. D. Gu, and T. Valla, Nat. Phys. 12, 550 (2016).

[5] Y. J. Wang E. Liu, H. Liu, Y. Pan, L. Zhang, J. Zeng, Y. Fu, M. Wang, K. Xu, and Z. Huang et al., Nat. Commun. 7, 13142 (2016).

[6] P. Li, Y. Wen, X. He, Q. Zhang, C. Xia, Z. M. Yu, S. A. Yang, Z. Y. Zhu, H. N. Alshareef, and X. X. Zhang, Nat. Commun. 8, 2150 (2017).

[7] B. Q. Lv, H. M. Weng, B. B. Fu, X. P. Wang, H. Miao, J. Ma, P. Richard, X. C. Huang, L. X. Zhao, and G. F. Chen et al., Phys. Rev. X 5, 031013 (2015).

[8] Z. K. Liu, B. Zhou, Y. Zhang, Z. J. Wang, H. M. Weng, D. Prabhakaran, S.-K. Mo, Z. X. Shen, Z. Fang, and X. Dai et al., Science 343, 864 (2014).

[9] S. Borisenko, Q. Gibson, D. Evtushinsky, V. Zabolotnyy, B. Buchner, and R. J. Cava, Phys. Rev. Lett. 113, 027603 (2014).

[10] P. J. W. Moll, N. L. Nair, T. Helm, A. C. Potter, I. Kimchi, A. Vishwanath, and J. G. Analytis, Nature (London) 535, 266 (2016).

[11] F. Arnold, C. Shekhar, S.-C. Wu, Y. Sun, R. D. dos Reis, N. Kumar, M. Naumann, M. O. Ajeesh, M. Schmidt, and A. G. Grushin et al., Nat. Commun. 7, 11615 (2016).

[12] R. D. dos Reis, M. O. Ajeesh, N. Kumar, F. Arnold, C. Shekhar, M. Naumann, M. Schmidt, M. Nicklas, and E. Hassinger, New J. Phys. 18, 085006 (2016).

[13] A. A. Burkov, Phys. Rev. B 96, 041110(R) (2017).

[14] S. Nandy, G. Sharma, A. Taraphder, and S. Tewari, Phys. Rev. Lett. 119, 176804 (2017).

[15] A. A. Burkov, Phys. Rev. Lett. 120, 016603 (2018).

[16] A. A. Burkov, Phys. Rev. B 97, 165104 (2018).
[17] N. Kumar, S. N. Guin, C. Felser, and C. Shekhar, Phys. Rev. B 98, 041103(R) (2018).

[18] H. Li, H. Wang, H. He, J. Wang, and S.-Q. Shen, Phys. Rev. B 97, 201110(R) (2018).

[19] P. Li, C. H. Zhang, J. W. Zhang, Y. Wen, and X. X. Zhang, Phys. Rev. B 98, 121108(R) (2018).

[20] M. Wu, G. Zheng, W. Chu, Y. Liu, W. Gao, H. Zhang, J. Lu, Y. Han, J. Zhou, and W. Ning et al., Phys. Rev. B 98, 161110(R) (2018).

[21] R. Singha, S. Roy, A. Pariari, B. Satpati, and P. Mandal, Phys. Rev. B 98, 081103(R) (2018).

[22] Y. Wang, J. X. Gong, D. D. Liang, M. Ge, J. R. Wang, W. K. Zhu, and C. J. Zhang, arXiv:1801.05929v2.

[23] D. D. Liang, Y. J. Wang, W. L. Zhen, J. Yang, S. R. Weng, X. Yan, Y. Y. Han, W. Tong, W. K. Zhu, and L. Pi et al., AIP Adv. 9, 055015 (2019).

[24] C.-H. Lee, E. C. Silva, L. Calderin, M. A. T. Nguyen, M. J. Hollander, B. Bersch, T. E. Mallouk, and J. A. Robinson, Sci. Rep. 5, 10013 (2015).

[25] M. Kim, S. Han, J. H. Kim, J.-U. Lee, Z. Lee, and H. Cheong, 2D Mater. 3, 034004 (2016).

[26] See Supplemental Material at http://link.aps.org/supplemental/ 10.1103/PhysRevB.100.205128 for the device fabrication details, SdH oscillations raw data, ordinary Hall effect, more angular dependent PHE at various magnetic fields, and more field dependent PHE, sample growth condition, and the fitting phase angle explanation. Temperature dependent PHE amplitude with other device thickness.

[27] C. Wang et al., Phys. Rev. B 94, 241119(R) (2016).

[28] A. A. Burkov, Phys. Rev. B 91, 245157 (2015).

[29] M. Udagawa and E. J. Bergholtz, Phys. Rev. Lett. 117, 086401 (2016).

[30] A. C. Potter, I. Kimchi, and A. Vishwanath, Nat. Commun. 5, 5161 (2014).

[31] C. Zhang, A. Narayan, S. Lu, J. Zhang, H. Zhang, Z. Ni, X. Yuan, Y. Liu, J.-H. Park, and E. Zhang et al., Nat. Commun. 8, 1272 (2017). 
[32] C. Zhang, Y. Zhang, X. Yuan, S. Lu, J. Zhang, A. Narayan, Y. Liu, H. Zhang, Z. Ni, and R. Liu et al., Nature (London) 565, 331 (2019).

[33] B. C. Lin, S. Wang, S. Wiedmann, J. M. Lu, W. Z. Zheng, D. Yu, and Z. M. Liao, Phys. Rev. Lett. 122, 036602 (2019).

[34] Z. W. Zhu, X. Lin, J. Liu, B. Fauque, Q. Tao, C. L. Yang, Y. G. Shi, and K. Behnia, Phys. Rev. Lett. 114, 176601 (2015).

[35] Z. Z. Du, C. M. Wang, H.-Z. Lu, and X. C. Xie, Phys. Rev. Lett. 121, 266601 (2018).
[36] K. Kang, T. Li, E. Sohn, J. Shan, and K. F. Mak, Nat. Mater. 18, 324 (2019).

[37] Q. Ma, S.-Y. Xu, H. Shen, D. MacNeill, V. Fatemi, T.-R. Chang, A. M. M. Valdivia, S. Wu, Z. Du, and C.-H. Hsu et al., Nature (London) 565, 337 (2019).

[38] V. Fatemi, Q. D. Gibson, K. Watanabe, T. Taniguchi, R. J. Cava, and P. Jarillo-Herrero, Phys. Rev. B 95, 041410(R) (2017).

[39] Y.-Y. Lv, X. Li, B.-B. Zhang, W. Y. Deng, S.-H. Yao, Y. B. Chen, J. Zhou, S.-T. Zhang, M.-H. Lu, and L. Zhang et al., Phys. Rev. Lett. 118, 096603 (2017). 